\title{
Clinical Aspects of Sentinel Lymph Node Biopsy in Gastric Cancer
}

\author{
Dezso Toth* \\ Department of General Surgery, University of Debrecen, Kenezy Gyula University Hospital, Hungary
}

Received: May 19, 2018; Published: May 29, 2018

*Corresponding author: Dezso Toth, Department of General Surgery, University of Debrecen, Kenezy Gyula University Hospital, 2-26 Bartok Street, Debrecen 4031, Hungary

\begin{abstract}
Forty percent of patients with gastric cancer are node negative, so they have an unnecessarily extended lymph node dissection with a higher rate of morbidity and mortality. Successful sentinel lymph node (SLN) mapping may help to reduce the number of extended lymphadenectomy. Sentinel lymph node biopsy (SNB) is indicated in patients with T1 or T2 tumors; primary lesions < than $4 \mathrm{~cm}$ in diameter; and clinical N0 gastric cancer. The injection method and selection of tracers for SLN mapping in gastric cancer remain controversial. However, the use of dual tracer and in cases with non-palpable lesions, the submucosal marking method is recommended. The identification rate and sensitivity are the highest in time performing SLN biopsy after dye injection $\geq 15 \mathrm{~min}$, the number of SLNs $\geq 5$ and application of the basin dissection. The cardinal problem in the SNB concept is the intraoperative false negative rate, the combination of hematoxylin-eosin and immunohistochemical staining has the highest sensitivity of intraoperative pathologic detection by frozen tissue sections.

The application of SNB has a beneficial side effect, as it significantly increased the number of harvested LN and the ratio of the number of the harvested LN per time, although an extensive surgical experience is necessary for application of SNB concept. If the SLN contains tumor deposit(s), extended dissection is warranted, but if findings are negative, the patient could be spared additional complications associated with extended dissection. Finally, the SNB could be suitable for tumors following endoscopic resection and could represent a new era of sentinel node navigation surgery in early gastric cancer. However, the clinical application of SLN biopsy should be limited to the patients of cT1N0M0 gastric cancer within the confines of prospective randomized trial.
\end{abstract}

Keywords: Gastric Cancer; Surgery; Lymphadenectomy; Sentinel Node Biopsy

\section{Introduction}

The modern, optimal treatment of patients with different neoplasms can be achieved, in most cases, with the stage adapted, combined modality therapy according to international protocols. In case of solid tumors the presence and exact number, or the absence of lymph node (LN) metastases are among the most important prognostic factors as in gastric cancer, where the presence of lymph node metastases is the most important predictive factor of survival following curative resection [1,2]. Moreover, a lot of study proved that LN metastasis is an independent risk factor for local recurrence as well as the time interval between radical gastrectomy and hepatic metastasis in patients after R0 resection [3,4]. Almost three hundred thousand patients with gastric adenocarcinoma do not have lymph node metastasis in the one million new cases each year $[5,6]$.

So, the one-third of patients with gastric adenocarcinoma has an unnecessarily extended lymph node dissection with a higher rate of morbidity and mortality. Preoperative imaging techniques provide a much more accurate determination of the $\mathrm{T}$ and $\mathrm{M}$ stage than that of the $\mathrm{N}$ stage. These preoperative diagnostic tools have a low sensitivity and specificity defining the patient subpopulation which would and which would not benefit from an extended lymph node dissection. For pathologic lymph node involvement the sensitivity, specificity and accuracy of spiral computer tomography is 73.1, 50.0 and $84.2 \%$, respectively [7] and endoscopic ultrasonography has an accuracy of $68.6 \%$, with a sensitivity and specificity of $66.7 \%$ and $73.7 \%$, respectively [8]. The real problem of these imaging procedures is that exclusion of endoscopic ultrasonography, only the size of the lymph nodes is taken into account.

In association with $\mathrm{T}$ stage, lymph node involvement can be found in $15 \%$ of patients with carcinoma confined to the mucosa, whereas lymph node metastases were detected in $23.4 \%, 48.2 \%$, and $69.8 \%$ of patients with carcinoma invading the submucosa, muscular is propria and serous layer, respectively [9]. Gertler showed that not only infiltration of the submucosa but also lymphatic vessel invasion, multifocal tumor growth, younger patient age and poor tumor differentiation were associated with nodal 
disease [10]. Besides T stage, lymph node involvement can also be influenced by tumor size. The overall accuracy of tumor size for preoperative N staging was $82.13 \%$ [11]. The incidence of lymph node metastasis in patients with a cancer size of $3-5 \mathrm{~cm}$ is $64.9 \%$, $80 \%$ in patients with a cancer size of $5-7 \mathrm{~cm}$ and $84.3 \%$ in patients with a cancer size of $>7 \mathrm{~cm}$ [9]. Additionally, early gastric cancer (EGC) has nodal metastases in $38.9 \%$ in poorly differentiated or undifferentiated types of tumor, in $41.7 \%$ with Lauren diffuse type and in $33.3 \%$ with a size larger than $3 \mathrm{~cm}$ [12]. Yang et al. [13] found that venous invasion, submucosal invasion or antral tumor location were independent predictors for LN metastasis in multivariate analysis. The rates of LN metastasis were $1.1 \%$ for patients with one or no predictor and $17.8 \%$ for those with two or more predictors [13].

The correct status of lymph node metastases can be obtained only by histology following an optimally extended node dissection. The removal of further lymph nodes on the other hand, increases operative time, the rate of complications, and if negative may be considered unnecessary. The three earliest studies found a higher morbidity and mortality rate following extended lymph node dissection of patients with gastric cancer when compared to those undergoing D1 dissection only [14-16]. These higher rates were related mostly to splenectomy and pancreatectomy. Moreover, extended LN dissection did not have any effect on oncological outcomes. The relapse risk and survival were similar in these studies. Only the revision of the Dutch trial showed better survival in advanced disease in the D2 group, after 11-year follow-up [17]. The 15-year follow-up results revealed that cancer-related death rates were lower $(37 \%$ versus $48 \%$ ) with a lower rate of local recurrence in the D2 lymphadenectomy group (Table 1) [18].

Table 1: Sensitivity and specificity of SLN mapping procedure in correlation to the marking method (submucosal versus subserosal) [33].

\begin{tabular}{|c|c|c|c|}
\hline & $\begin{array}{c}\text { Submucosal } \\
(\mathbf{n = 2 0})\end{array}$ & $\begin{array}{c}\text { Subserosal } \\
(\mathbf{n = 3 7 )}\end{array}$ & $\begin{array}{c}\text { Result } \\
(\mathbf{9 5 \%} \mathbf{C I})\end{array}$ \\
\hline Mean number of SLN & 4.3 & 5.4 & \\
\hline Detection rate (\%) & $100 \%$ & $97.3 \%$ & equivalent \\
\hline False negative rate (\%) & $0 \%$ & $5.9 \%$ & \\
\hline Sensitivity (\%) & $100 \%$ & $94.1 \%$ & equivalent \\
\hline Specificity (\%) & $100 \%$ & $100 \%$ & equivalent \\
\hline
\end{tabular}

Criterion of equivalence: $95 \%$ of confidence interval (CI) should be fully within the range $0.8-1.25$

The two latest randomized trials from the 21st century did not present significant differences in postoperative mortality between the D1 and D2 group [19,20]. The morbidity rate was higher with D2 lymphadenectomy in the Taiwanese trial (which compared D1 to D3 dissection; however their D3 lymphadenectomy is similar to the current definition of D2 dissection). The Italian study did not show this difference and proved that D2 dissection could be performed safely without splenectomy and distal pancreatectomy, with comparable mortality and morbidity to those with D1 dissection in specialized centers $[20,21]$. These rates are comparable to the Japan Clinical Oncology Group (JCOG) 9501 trial and the nationwide Japanese registry where the mortality was less than $2 \%$ after D2 dissection $[22,23]$. Neither did the latter study find any survival benefit from the extended lymphadenectomy [24]. These facts prove that the adequate extension of lymphadenectomy is controversial, even now. Successful sentinel lymph node (SLN) mapping may help to define those patients who would and those who would not benefit from an extended lymph node dissection in association with gastrectomy.

\section{Technical Aspects of Sentinel Lymph Node Biopsy \\ Sentinel Lymph Node Detection Techniques}

The first potentially affected lymph node, the sentinel lymph node, reliably reflects the status of the nodes in the second and third line, which is supported by data of numerous publications. The concept of sentinel lymph node biopsy (SNB) can determine the existence of LN metastases intraoperatively. This is a multistep technique consisted of six essential elements: indication, the selection of a tracer, a proper tracer injection method, the objective detection of SLNs, a reliable biopsy technique, and the precise detection of nodal metastasis [25].

Endoscopic and minor surgical gastric resections with D1 or modified D1 lymphadenectomies increased in EGC significantly. These minor procedures have been proved to have improved postoperative quality of life, while maintaining 5-year survival rates $>95 \%$ [26]. In general, SLN mapping and biopsy is indicated in patients with T1 or T2 tumors; primary lesions $<$ than $4 \mathrm{~cm}$ in diameter; and clinical N0 gastric cancer. [27]. The injection method and selection of tracers for SLN mapping in gastric cancer remain controversial. Studies from Japan and from Western Europe have demonstrated a high detection rate ( $>95 \%$ ) and a sensitivity of 90 95 percent [28-32]. Some authors use dye alone (patent blue, indocyanine green (ICG), isosulfan blue [31,33-35], while others 99m Tc colloid [28], and uses the combination of these tracers [36].

As reported by a meta-analysis, radioactive isotope alone got a pooled identification rate and sensitivity of 92.1 and $76.4 \%$ while dye alone was 92.1 and $72.7 \%$ [37]. The latest systematic review concluded that the use of dual tracer in SLN mapping got the highest identification rate and sensitivity (97.0 and 89.0\%) [38]. There are some experimental results with carbon nanoparticles with promising results [39] and with the novel ICG fluorescence systems such as the D-light P system, the technique can be used in the room light and the laparoscopic surgery [38].

Keeping the well-known differences between Asian and European gastric cancer phenotypes in mind [40-42], Toth et al. have evaluated, for the first time, the SLN mapping using the blue dye only method in Hungary. After laparotomy, the lesser sac was opened (if it was needed) and 4 X $0.5 \mathrm{~mL}$ blue dye (Bleu Patente V Sodique Guerbet 2,5\%, F-95943 Roissy CdG, Cedex, France) was injected in four quadrants around the tumor. Lymphatic drainage was observed for up to 15 minutes following injection. Blue stained lymph nodes were marked as sentinel lymph nodes and D2 lymph node dissection was performed after gastric resection or total gas- 
trectomy [33]. In this investigation only the negative sentinel nodes were examined for micro metastasis, because of the widely recognized concept that lymphatic micro metastasis spread first to sentinel nodes in gastric cancer [43].

According to the marking techniques, earlier Yaguchi and Lee have compared the subserosal to the submucosal labeling method without any significant difference and they suggest the endoscopic injection of a tracer in cases of non-palpable tumors and/or laparoscopic procedures $[44,45]$. In the Hungarian study the submucosal and subserosal marking method (Figures 1 \& 2) was proven equivalent in detection rate, sensitivity and specificity based on $95 \%$ confidence interval (CI) of ratio of indicators (Table 1) [33]. It is eminent that body-mass index (BMI) affects the sentinel lymph node detection rate [46]. The latter mentioned study proved that the identification of sentinel lymph nodes in obese patients can be difficult owing to the feathering of blue dye in the fatty tissue. This was concluded as the only patient in whom marking did not occur had a BMI significantly higher than average (26.8 versus 22.8 ) [33]. To avoid quick dispersal to multiple lymph nodes Kong applied ICG/poly- $\gamma$-glutamic acid (PGA) complex, which remained longer than diluted ICG in animal model [47].

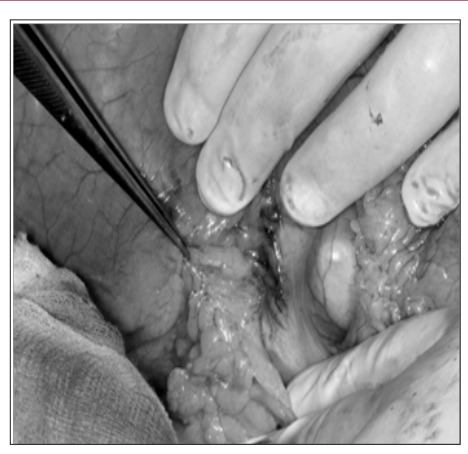

Figure 1: Sentinel lymph node mapping following submucosal marking by an endoscopist.

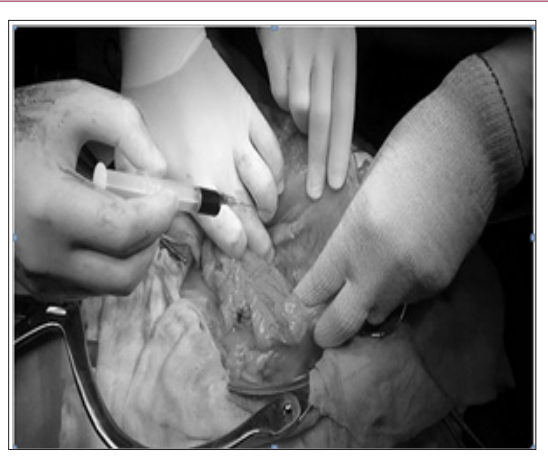

Figure 2: Subserosal labelling by a surgeon.

Latest meta-analysis proved when considering the time performing SLN biopsy after dye injection, the pooled identification rate and sensitivity of the $\geq 15$ min subgroup was much higher than that of the $<15$ min subgroup ( 97.3 vs $96.2 \%, 95.2$ vs $81.0 \%$ ). The number of SLNs, the pooled sensitivity of the $\geq 5$ subgroup was much higher than that of the $<5$ subgroup ( 94.4 vs $83.9 \%$ ) and the methods of SLN biopsy, the pooled sensitivity of the basin dissection subgroup was much higher than that of the pick-up subgroup ( 95.9 vs $86.9 \%$ ). Moreover, the identification rate of the opening surgery was a little higher than that of the laparoscopic subgroup (97.6 vs93.9\%) [38]. Besides this, regarding the countries where researches were conducted, the pooled identification rate and sensitivity of the Japan subgroup was a little higher than that of the not in Japan subgroup (98.0 vs $93.4 \%, 89.6$ vs $87.3 \%$ ) and in concern of publication year, the identification rate and sensitivity of the $\geq$ 2013 subgroup was higher than that of the $<2013$ subgroup (97.6 vs $95.5 \%, 90.5$ vs $86.4 \%$ ) [38].

\section{Sentinel Lymph Node Detection Failure and Evaluation Techniques}

The cardinal problem in the SNB concept is the intraoperative false negative rate. The JCOG 0302 trial called attention to the importance of the learning curve and the inadequacy of the pick-up method. The demand for only five patients per institute provided an insufficient learning period which presented a $46 \%$ false negative rate [48]. Miyashiro demonstrated that an extensive surgical experience is necessary for application of SNB concept [49] and standardization of SLN mapping technique, using improved tracer, and guideline to evaluate the positiveness of SLN specimen should be planned to incorporate SNB in routine practice [50]. On the other hand, in a Japanese multicenter trial, it was found that $\sim 25 \%$ of patients with SN metastases that were diagnosed using permanent sections could not be identified using H-E staining of frozen sections collected intraoperatively [51]. Moreover, the sensitivity of intraoperative pathologic detection by frozen tissue sections into account, the pooled sensitivity of HE alone is $84.5 \%$. And this is lower than the HE and IHC combined subgroup whose pooled sensitivity is $86.4 \%$ [38].

Multistep level sections, immunohistochemistry [52,53], reverse transcription polymerase chain reaction (RT-PCR) [54-57], and the one-step nucleic acid amplification assay (OSNA) [55], have all been developed to reduce the false-negative rates and provide reliable diagnostic tools for micro metastases in SLN. This latter technique (OSNA) is possible to reduce the detection time to $\sim 30$ min [58] and this test has similar results to postoperative 2-mm-interval histological examination for the intraoperative diagnosis of LN metastasis [59]. Shimizu et al. [57] developed a more sensitive real-time PCR method by using cytokeratin 19 mRNA, cytokeratin 20 mRNA, and carcinoembryonic antigen (CEA) mRNA. They have reported that approximately $30 \%$ of patients with pathologically negative LNs were positive for RT-PCR.

\section{Advantages and Complications of Sentinel Lymph Node Biopsy}

The application of SNB has a beneficial side effect, as it significantly increased the number of harvested LN and the ratio of the number of the harvested LN per time [60]. During the Hungarian investigation any side-effect or complication with the blue dye method was not realized [33]. 


\section{Management of Positive And Negative Sentinel Lymph Node Biopsy}

If the SLN contains tumor deposit(s), extended dissection is warranted, but if findings are negative, the patient could be spared additional complications associated with extended dissection. Based on the results of the largest prospective multi-center trial from Japan with an identification rate of $97.5 \%$ and an accuracy of 99\% [51], a phase III multi-center trial for individualized surgery for EGC based on SLN mapping has been commenced in the Eastern Asian countries. The long-term results of these studies will be available between 2018 and 2020 .

\section{Conclusion}

Dutch and British prospective randomized trials found a higher morbidity and mortality rate following extended lymph node dissection of patients with gastric cancer when compared to those undergoing D1 dissection only $[15,16]$. The preoperative diagnostic tools have a low sensitivity and specificity defining the patient subpopulation which would and which would not benefit from an extended lymph node dissection. Recent studies and the latest meta-analysis of SLN mapping have shown a high detection rate (93.7\%) and an accuracy of 92\% [37] and suggest that the SNB concept could be suitable for tumors following endoscopic resection [61] and could represent a new era of sentinel node navigation surgery in early gastric cancer $[62,63]$. Various mapping techniques have been described in the literature. The injection method and selection of tracers for SLN mapping remain controversial. However, the equivalence between the submucosal marking and the subserosal injection was proved. In cases of non-palpable tumors, the endoscopical submucosal injection is the preffered technique. Finally, the clinical application of SLN biopsy should be limited to the patients of cT1N0M0 gastric cancer within the confines of prospective randomized trials.

\section{References}

1. Siewert JR, Bottcher K, Stein HJ, Roder JD (1998) Relevant prognostic factors in gastric cancer: ten-year results of the German Gastric Cancer Study. Ann Surg 228: 449-461.

2. Kim JP, Kim YW, Yang HK, Noh DY (1994) Significant prognostic factors by multivariate analysis of 3926 gastric cancer patients. World J Surg 18: 872-877.

3. Deng JY, Liang H, Sun D, Zhan HJ, Zhang RP (2008) Analysis of risk factors for the interval time, number and pattern of hepatic metastases from gastric cancer after radical gastrectomy. World J Gastroenterol 14: 24402447.

4. Kodera Y, Yamamura Y, Shimizu Y, Torii A, Hirai T, et al. (1998) Lymph node status assessment for gastric carcinoma: is the number of metastatic lymph nodes really practical as a parameter for $\mathrm{N}$ categories in the TNM Classification? Tumor Node Metastasis. J Surg Oncol 69: 1520.

5. Jemal A, Bray F, Center MM, Ferlay J, Ward E, et al. (2011) Global cancer statistics. CA Cancer J Clin 61: 69-90.

6. Kooby DA, Suriawinata A, Klimstra DS, Brennan MF, Karpeh MS (2003) Biologic predictors of survival in node-negative gastric cancer. Ann Surg 237: 828-835.

7. Chamadol N, Wongwiwatchai J, Bhudhisawasd V, Pairojkul C (2008) Accuracy of spiral CT in preoperative staging of gastric carcinoma: correlation with surgical and pathological findings. J Med Assoc Thai 3: 356-363.

8. Xi WD, Zhao C, Ren GS (2003) Endoscopic ultrasonography in preoperative staging of gastric cancer: determination of tumor invasion depth, nodal involvement and surgical resectability. World J Gastroenterol 9: 254-257.

9. Zuo CH, Xie H, Liu J, Qiu XX, Lin JG, et al. (2014) Characterization of lymph node metastasis and its clinical significance in the surgical treatment of gastric cancer. Mol Clin Oncol 2: 821-826.

10. Gertler R, Stein HJ, Schuster T, Rondak IC, Hofler H, et al. (2014) Prevalence and topography of lymph node metastases in early esophageal and gastric cancer. Ann Surg 259: 96-101.

11. Huang CM, Xu M, Wang JB, Zheng $\mathrm{CH}$, et al. (2014) Is tumor size a predictor of preoperative $\mathrm{N}$ staging in $\mathrm{T} 2-\mathrm{T} 4 \mathrm{a}$ stage advanced gastric cancer? Surg Oncol 23: 5-10.

12. Bravo Neto GP, dos Santos EG, Victer FC, Carvalho CE (2014) Lymph node metastasis in early gastric cancer. Rev Col Bras Cir 41: 11-17.

13. Yang HJ, Kim SG, Lim JH, Choi J, Im JP, et al. (2015) Predictors of lymph node metastasis in patients with non-curative endoscopic resection of early gastric cancer. Surg Endosc 29: 1145-1155.

14. Dent DM, Madden MV, Price SK (1988) Randomized comparison of R1 and R2 gastrectomy for gastric carcinoma. Br J Surg 75: 110-112.

15. Cuschieri A, Fayers P, Fielding J, Craven J, Bancewicz J, et al. (1996) Postoperative morbidity and mortality after D1 and D2 resections for gastric cancer: preliminary results of the MRC randomised controlled surgical trial. The Surgical Cooperative Group. Lancet 347: 995-999.

16. Bonenkamp JJ, Songun I, Hermans J, Sasako M, Welvaart K, et al. (1995) Randomised comparison of morbidity after D1 and D2 dissection for gastric cancer in 996 Dutch patients. Lancet 345: 745-748.

17. Bonenkamp JJ, Hermans J, Sasako M, van de Velde CJ, Welvaart K, et al. (1999) Dutch Gastric Cancer Group. Extended lymph-node dissection for gastric cancer. N Engl J Med 340: 908-914.

18. Songun I, Putter H, Kranenbarg EM, Sasako M, van de Velde CJ (2010) Surgical treatment of gastric cancer: 15-year follow-up results of the randomised nationwide Dutch D1D2 trial. Lancet Oncol 11: 439-449.

19. Degiuli M, Sasako M, Ponti A, Italian Gastric Cancer Study Group (2010) Morbidity and mortality in the Italian Gastric Cancer Study Group randomized clinical trial of D1 versus D2 resection for gastric cancer. $\mathrm{Br}$ J Surg 97: 643-649.

20. Wu CW, Hsiung CA, Lo SS, Hsieh MC, Shia LT, et al. (2004) Randomized clinical trial of morbidity after D1 and D3 surgery for gastric cancer. Br J Surg 91: 283-287.

21. Jiang L, Yang KH, Chen Y, Guan QL, Zhao P, et al. (2014) Systematic review and meta-analysis of the effectiveness and safety of extended lymphadenectomy in patients with resectable gastric cancer. Br J Surg 101: 595-604.

22. Sano T, Sasako M, Yamamoto S, Nashimoto A, Kurita A, et al. (2004) Gastric cancer surgery: morbidity and mortality results from a prospective randomized controlled trial comparing D2 and extended para-aortic lymphadenectomy--Japan Clinical Oncology Group study 9501. J Clin Oncol 22: 2767-2773.

23. Fujii M, Sasaki J, Nakajima T (1999) State of the art in the treatment of gastric cancer: from the 71st Japanese Gastric Cancer Congress. Gastric Cancer 2: 151-157.

24. Degiuli M, Sasako M, Ponti A, Vendrame A, Tomatis M, et al. (2014) Randomized clinical trial comparing survival after D1 or D2 gastrectomy for gastric cancer. Br J Surg 101: 23-31.

25. Kinami S, Kosaka T (2017) Laparoscopic sentinel node navigation surgery for early gastric cancer. Transl Gastroenterol Hepatol 2: 42. 
26. Jeong O, Ryu SY, Park YK (2009) Accuracy of surgical diagnosis in detecting early gastric cancer and lymph node metastasis and its role in determining limited surgery. J Am Coll Surg 209: 302-307.

27. Lianos GD, Hasemaki N, Vaggelis G, Anastasia Karampa, Zoi Anastasiadi, et al. (2016) Sentinel node navigation in gastric cancer: new horizons for personalized minimally invasive surgical oncology? Translational Gastroenterology and Hepatology 1: 91.

28. Kitagawa Y, Fujii H, Mukai M, Kubota T, Otani Y, et al. (2002) Radioguided sentinel node detection for gastric cancer. Br J Surg 89: 604-608.

29. Ichikura T, Morita D, Uchida T, Okura E, Majima T, et al. (2002) Sentinel node concept in gastric carcinoma. World J Surg 26: 318-322.

30. Hiratsuka M, Miyashiro I, Ishikawa O, Furukawa H, Motomura K, et al. (2001) Application of sentinel node biopsy to gastric cancer surgery. Surgery 129: 335-340.

31. Rino Y, Takanashi Y, Hasuo K, Kawamoto M, Ashida A, et al. (2007) The validity of sentinel lymph node biopsy using dye technique alone in patients with gastric cancer. Hepatogastroenterology 54(78): 18821886.

32. Gretschel S, Bembenek A, Ulmer $\mathrm{Ch}, \mathrm{Hu}$ "nerbein $\mathrm{M}$, Markwardt J, et al (2005) Prediction of gastric cancer lymph node status by sentinel lymph node biopsy and the Maruyama computer model. Eur J Surg Oncol 31(4): 393-400.

33. Toth D, Kincses Z, Plosz J, Torok M, Kovacs I, et al. (2011) Value of sentinel lymph node mapping using a blue dye-only method in gastric cancer: a single-center experience from North-East Hungary. Gastric Cancer 14: 360-364.

34. Tajima Y, Yamazaki K, Masuda Y, Kato M, Yasuda D, et al. (2009) Sentinel node mapping guided by indocyanine green fluorescence imaging in gastric cancer. Ann Surg 249: 58-62.

35. Ryu KW, Lee JH, Kim HS, Kim YW, Choi IJ, et al. (2003) Prediction of lymph nodes metastasis by sentinel node biopsy in gastric cancer. Eur J Surg Oncol 29: 895-899.

36. Aikou T, Higashi H, Natsugoe S, Hokita S, Baba M, et al. (2001) Can sentinel node navigation surgery reduce the extent of lymph node dissection in gastric cancer? Ann Surg Oncol 8: 90S-93S.

37. Wang Z, Dong ZY, Chen JQ Liu JL (2012) Diagnostic value of sentinel lymph node biopsy in gastric cancer: a meta-analysis. Ann Surg Oncol 19: $1541-1550$.

38. Huang L, Wei T, Chen J, Zhou D (2017) Feasibility and diagnostic performance of dual-tracer-guided sentinel lymph node biopsy in cT1-2N0M0 gastric cancer: a systematic review and meta-analysis of diagnostic studies. World Journal of Surgical Oncology 15: 103.

39. Yan J, Zheng X, Liu Z, Yu J, Deng Z, et al. (2016) A multicenter study of using carbon nanoparticles to show sentinel lymph nodes in early gastric cancer. Surg Endosc 30(4): 1294-1300.

40. Hohenberger P, Gretschel S (2003) Gastric cancer. Lancet 362: 305-315.

41. La Vecchia CL, Negri E, Franceschi S, Gentile A, et al. (1992) Family history and the risk of stomach and colorectal cancer. Cancer 70: 50-55.

42. Lauren P (1965) The two histological main types of gastric carcinoma: diffuse and so-called intestinal-type carcinoma. An attempt at histoclinical classification. Acta Pathol Microbiol Scand 64: 31-39.

43. Ishii K, Kinami S, Funaki K, Fujita H, Ninomiya I, et al. (2008) Detection of sentinel and non-sentinel lymph node micro metastases by complete serial sectioning and immunohistochemical analysis for gastric cancer. J Exp Clin Cancer Res 27(1): 7.

44. Yaguchi Y, Ichikura T, Ono S, Tsujimoto H, Sugasawa H, et al. (2008) How should tracers be injected to detect for sentinel nodes in gastric cancersubmucosally from inside or subserosally from outside of the stomach? J Exp Clin Cancer Res 27: 79.
45. Lee JH, Ryu KW, Kim CG, Kim SK, Choi IJ, et al. (2005) Comparative study of the subserosal versus submucosal dye injection method for sentinel node biopsy in gastric cancer. Eur J Surg Oncol 31: 965-968.

46. Nakahara T, Kitagawa Y, Yakeuchi H, Fujii H, Suzuki T, et al. (2008) Preoperative lymphoscintigraphy for detection of sentinel lymph node in patients with gastric cancer--initial experience. Ann Surg Oncol 15: 1447-1453.

47. Kong SH, Noh YW, Suh YS, Park HS, Lee HJ, et al. (2015) Evaluation of the novel near-infrared fluorescence tracers pullulan polymer nanogel and indocyanine green $/ \gamma$-glutamic acid complex for sentinel lymph node navigation surgery in large animal models. Gastric Cancer 18: 55-64.

48. Miyashiro I, Hiratsuka M, Sasako M, Sano T, Mizusawa J, et al. (2014) Gastric Cancer Surgical Study Group (GCSSG) in the Japan Clinical Oncology Group (JCOG). High false-negative proportion of intraoperative histological examination as a serious problem for clinical application of sentinel node biopsy for early gastric cancer: final results of the Japan Clinical Oncology Group multicenter trial JCOG0302. Gastric Cancer 17: 316-323.

49. Miyashiro I, Hiratsuka M, Kishi K, Takachi K, Yano M, et al. (2013) Intraoperative diagnosis using sentinel node biopsy with indocyanine green dye in gastric cancer surgery: an institutional trial by experienced surgeons. Ann Surg Oncol 20: 542-546.

50. Yashiro M, Matsuoka T (2015) Sentinel node navigation surgery for gastric cancer: Overview and perspective. World J Gastrointest Surg 7: $1-9$.

51. Kitagawa Y, Takeuchi H, Takagi Y, Shoji Natsugoe, Masanori Terashima, et al. (2013) Sentinel node mapping for gastric cancer: a prospective multicenter trial in Japan. J Clin Oncol 31: 3704-3710.

52. Ishii K, Kinami S, Funaki K, Nishimura G, Kayahara M, et al. (2008) Detection of sentinel and non-sentinel lymph node micrometastases by complete serial sectioning and immunohistochemical analysis for gastric cancer. J Exp Clin Cancer Res 27: 7.

53. Uenosono Y, Natsugoe S, Ehi K, Hokita S, Aikou T, et al. (2005) Detection of sentinel nodes and micrometastases using radioisotope navigation and immunohistochemistry in patients with gastric cancer. Br J Surg 92: 886-889.

54. Ajisaka H, Miwa K (2003) Micrometastases in sentinel nodes of gastric cancer. Br J Cancer 89: 676-680.

55. Takeuchi H, Ueda M, Oyama T, Shimizu Y, Kitagawa Y, et al. (2010) Molecular diagnosis and translymphatic chemotherapy targeting sentinel lymph nodes of patients with early gastrointestinal cancers. Digestion 82: 187-191.

56. Arigami T, Natsugoe S, Uenosono Y, Shuichi Hokita, Takashi Aikou, et al. (2006) Evaluation of sentinel node concept in gastric cancer based on lymph node micro metastasis determined by reverse transcriptionpolymerase chain reaction. Ann Surg 243: 341-347.

57. Shimizu Y, Takeuchi H, Sakakura Y, Kitajima M, Kitagawa Y, et al. (2012) Molecular detection of sentinel node micrometastases in patients with clinical N0 gastric carcinoma with real-time multiplex reverse transcription-polymerase chain reaction assay. Ann Surg Oncol 19: 469477.

58. Yanagita S, Natsugoe S, Uenosono Y (2008) Detection of micrometastases in sentinel node navigation surgery for gastric cancer. Surg Oncol 17: 203-210.

59. Kumagai K, Yamamoto N, Miyashiro I, Tomita Y, Katai H, et al. (2014) Multicenter study evaluating the clinical performance of the OSNA assay for the molecular detection of lymph node metastases in gastric cancer patients. Gastric Cancer 17: 273-280.

60. Aoyama T, Fujikawa H, Cho H, Ogata T, Shirai J, et al. (2015) A methylene blue-assisted technique for harvesting lymph nodes after radical surgery for gastric cancer: a prospective, randomized, controlled study. Am J Surg Pathol 39: 266-273. 
61. Mayanagi S, Takeuchi H, Kamiya S, Niihara M, Nakamura R, et al. (2014) Suitability of sentinel node mapping as an index of metastasis in early gastric cancer following endoscopic resection. Ann Surg Oncol 21(9): 2987-2993.

62. Wang L, Ren W, Fan CQ Li YH, Zhang X, et al. (2011) Full-thickness endoscopic resection of nonintracavitary gastric stromal tumors: a novel approach. Surg Endosc 25(2): 641-647.

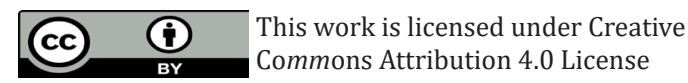

Submission Link: https://biomedres.us/submit-manuscript.php
63. Kong SH, Diana M, Liu YY, Lee HJ, Legner A, et al. (2015) Novel method for hybrid endo-laparoscopic full-thickness gastric resection using laparoscopic transgastric suture passer device. Surg Endosc 30: 16831691.

Assets of Publishing with us
RESEARCHES
- Global archiving of articles
- Immediate, unrestricted online access
- Rigorous Peer Review Process
https://biomedres.us/

